\title{
Complete Laryngeal Vestibular Closure
}

National Cancer Institute

\section{Source}

National Cancer Institute. Complete Laryngeal Vestibular Closure. NCI Thesaurus. Code C127254.

A finding of complete laryngeal vestibular closure; no air/contrast in laryngeal vestibule. 